\title{
Frontal Fibrosing Alopecia Coexisting with Lupus Erythematosus: Poor Response to Hydroxychloroquine
}

\author{
Letícia Arsie Contin Elisa Raquel Martins da Costa Marques Leandro Noriega \\ Dermatology Clinic, Hospital do Servidor Público Municipal de São Paulo, São Paulo, Brazil
}

\section{Established Facts}

- Frontal fibrosing alopecia is a primary lymphocytic cicatricial alopecia, considered a clinical variant of lichen planopilaris.

- Hydroxychloroquine is a treatment option for lichen planopilaris/frontal fibrosing alopecia and lupus erythematosus due to an antilymphocytic effect.

\section{Novel Insights}

- The association of frontal fibrosing alopecia and lupus erythematosus shows they possibly have a common immune background.

- We describe 4 cases of this disease association, with progressive frontal fibrosing alopecia despite treatment with hydroxychloroquine.

\section{Keywords}

Cicatricial alopecia · Frontal fibrosing alopecia .

Hydroxychloroquine · Lupus erythematosus

\begin{abstract}
Lupus erythematosus, especially the discoid form, and lichen planopilaris may be associated and can occur in different topographies (coexistence) or in the same lesion (lupus eythematosus/lichen planus overlap syndrome). Frontal fibrosing alopecia is considered a variant form of lichen
\end{abstract}

planopilaris and is characterized by frontotemporal hairline and eyebrow involvement. Of the association with lupus erythematosus we have only a few descriptions. Hydroxychloroquine and chloroquine diphosphate are antimalarial drugs described as viable treatment options for both diseases, due to an antilymphocytic effect. The association between frontal fibrosing alopecia and lupus erythematosus (discoid or systemic) is reported in this article, showing a progressive alopecia in the frontotemporal hairline despite treatment with hydroxychloroquine.

(c) 2016 S. Karger AG, Basel

\section{KARGER}

E-Mail karger@karger.com www.karger.com/sad
C 2016 S. Karger AG, Basel

2296-9195/16/0024-0162\$39.50/0
Leandro Noriega

Dermatology Clinic

Hospital do Servidor Público Municipal de São Paulo

60 Castro Alves, São Paulo, SP 01532000 (Brazil)

E-Mail leandronorieg@gmail.com 


\section{Introduction}

Discoid lupus erythematosus (DLE) and lichen planopilaris (LPP) are considered primary cicatricial alopecias and they are characterized by lymphocytic infiltrate $[1,2]$. DLE lesions may be similar to LPP as a patchy cicatricial alopecia. Frontal fibrosing alopecia (FFA) is considered a clinical variant of LPP, with clinical differences, characterized by alopecia in the frontotemporal hairline and eyebrows, sometimes associated with facial papules and hair loss on the limbs, the axillary and pubic regions, and loss of eyelashes [3-7].

The association of lupus erythematosus (LE) and LPP has been reported to coexist when two lesions are diagnosed, each of them with each of the conditions, or when both conditions occur simultaneously (clinical presentation and histological or immunohistochemical features of both diseases) in the same lesion, in which case it is called LE/lichen planus overlap syndrome $[8,9]$. The association of LE and FFA has also been described [7, 10-12].

The disease's activity is sometimes hard to define. Hydroxychloroquine efficacy for LPP/FFA has been proposed through the analysis of an index called LPP Activity Index, created to try to determine whether the disease is active or not based on erythema, perifollicular erythema, perifollicular scale, pruritus, burning sensation, pain, anagen hair at pull test, and progression [13].
Hydroxychloroquine and chloroquine diphosphate are considered as treatment options for both LE and LPP/ FFA [14]. They act by lowering the upregulated expression of regulatory T cells [15]. This is a report of 4 disease association cases and their response to hydroxychloroquine.

\section{Case Reports}

Case 1

A 55-year-old female patient presented with clinical and histopathological characteristics of DLE since 1987, with cutaneous lesions in the malar region, nasal dorsum, and lower lip. Antinuclear antibody (ANA) test was positive at $1 / 320$, without other criteria for systemic lupus. Upon this result, chloroquine treatment was then started. In 2008, she was diagnosed with FFA, despite her previous treatment. Both diseases are still active, with cutaneous lesions and significant progression of hair loss since diagnosis (Fig. 1).

\section{Case 2}

A 48-year-old female patient presented with DLE (Fig. 2) and Raynaud phenomenon since 1996. She has been intermittently treated with hydroxychloroquine since 2010. ANA test was negative and anti-Ro/SSA antibody was positive. She was diagnosed with FFA in 2013. DLE lesions are under control after treatment with hydroxychloroquine, but FFA is still active, with presence of perifollicular erythema, perifollicular scale, progression, and positive anagen pull test (Fig. 3a, b).
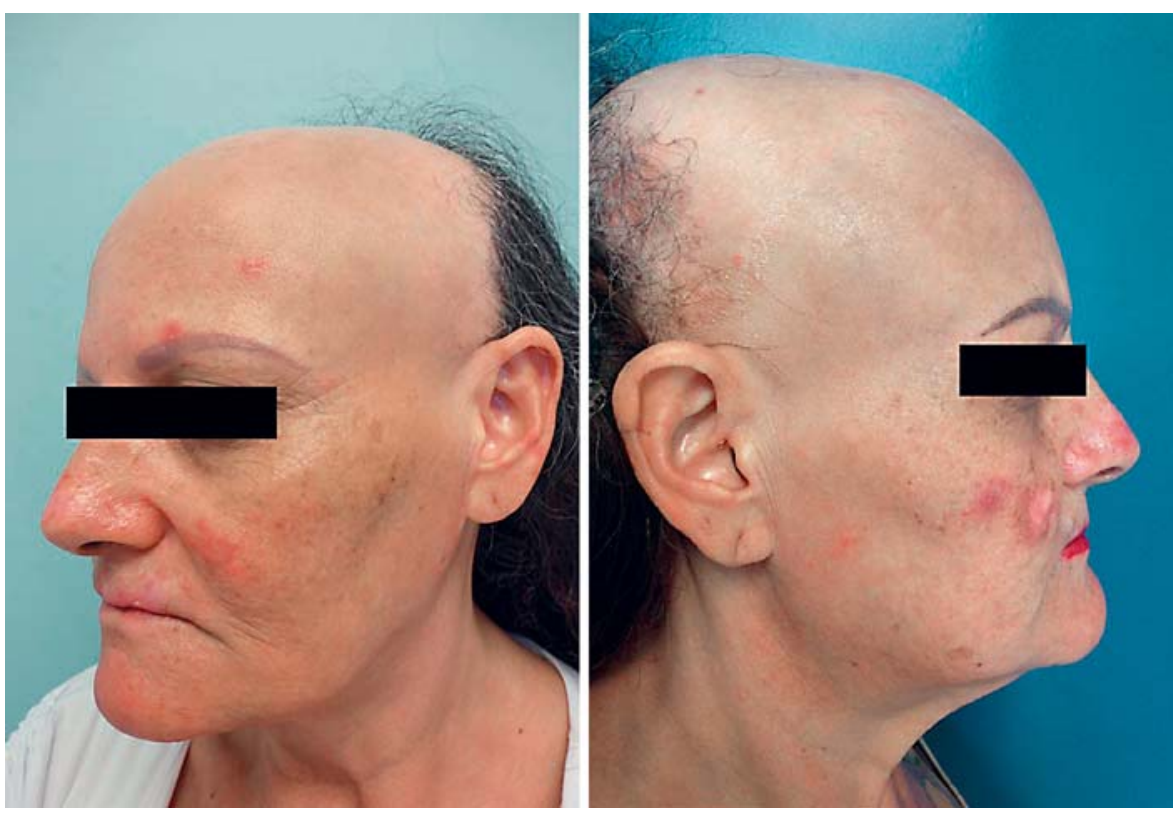

Fig. 1. Active discoid lupus erythematosus lesions associated with extensive frontal fibrosing alopecia.

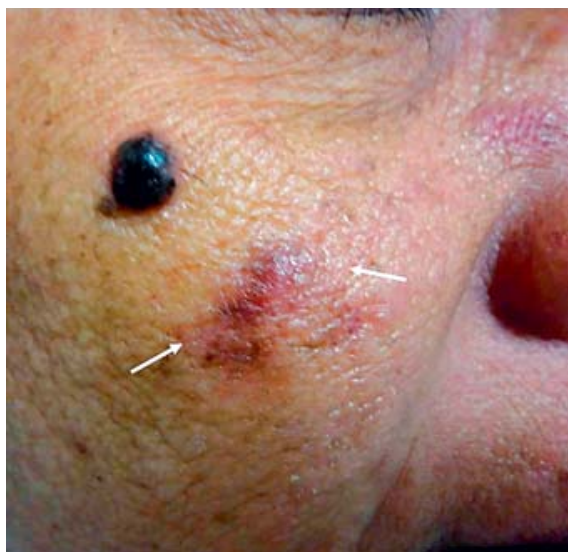

Fig. 2. Previous discoid lupus erythematosus lesion (right malar region). 
Fig. 3. a Stable discoid lupus and active frontal fibrosing alopecia. b Trichoscopy: frontal hairline with perifollicular scale.
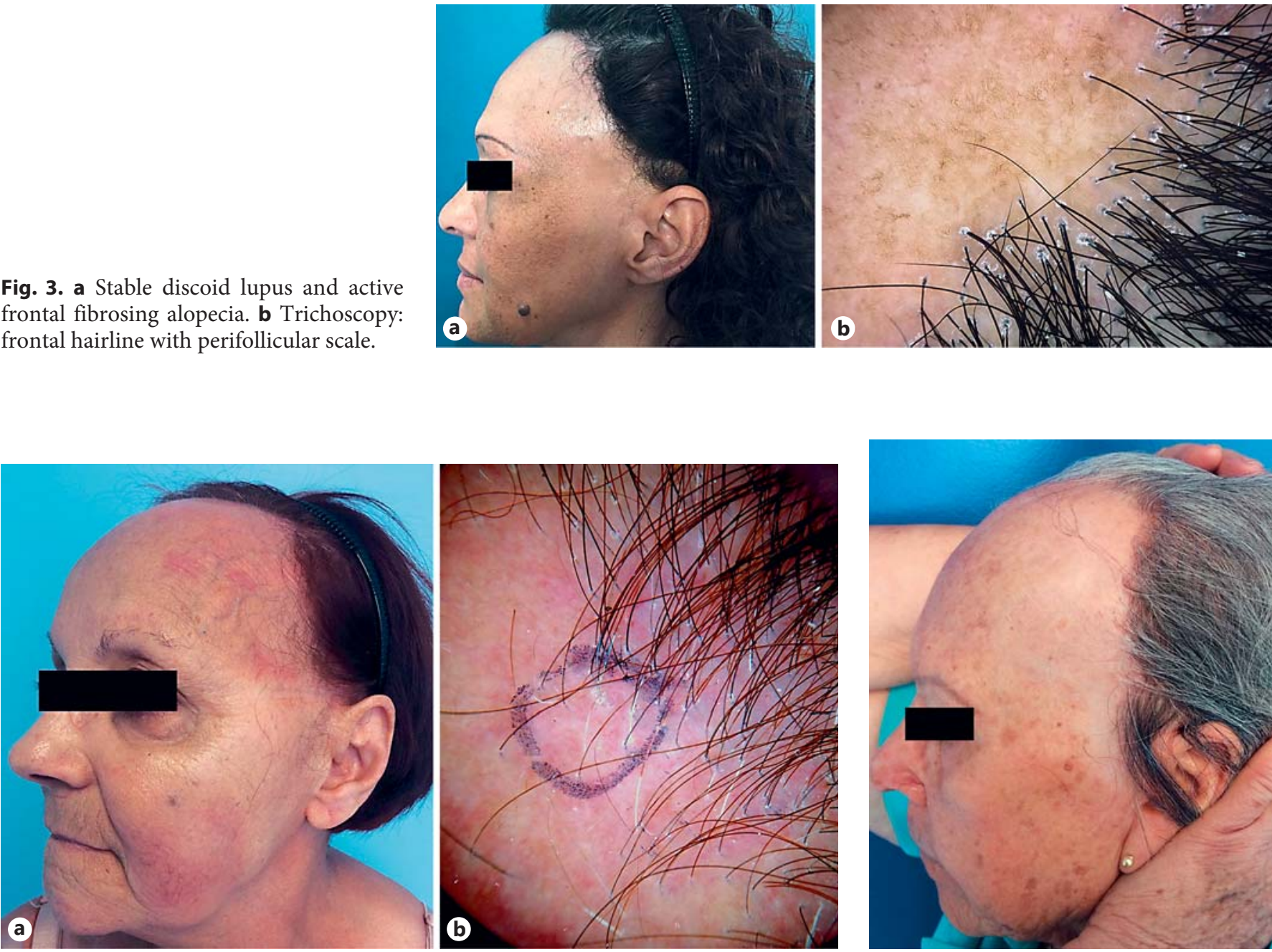

Fig.4. a Active discoid lupus and frontal fibrosing alopecia. b Trichoscopy: frontal hairline with erythema and perifollicular scale.

Fig. 5. Progressive frontal fibrosing alopecia.
Case 3

A 62-year-old female patient presented with systemic lupus erythematosus (SLE) since 2004, manifested as malar rash, arthralgia, polyadenomegaly, persistent anemia, positive ANA test at $1 / 1,280$, and low titers of C3 and C4. She was diagnosed with FFA in 2009, which is still active after 3 years of treatment with hydroxychloroquine, with perifollicular scale and positive progression in global photography (Fig. 4a, b).

\section{Case 4}

A 77-year-old female patient had been diagnosed with SLE approximately 35 years ago. After control of lupus activity, she maintained only the use of hydroxychloroquine since 2012 and despite this, developed FFA in 2014. Currently, SLE is under control, but FFA is still active and shows erythema, pruritus, and progression (Fig. 5).

\section{Discussion}

There are only a few descriptions of FFA and LE, with DLE being the most common association $[8,9,16]$. del Rei et al. [9] described FFA and DLE in 7 patients. Banka et al. [17] described FFA associated with DLE in 2 cases and FFA associated with SLE in 2 other cases. We also found the association of FFA with DLE in 2 patients and FFA with SLE in 2 other cases.

FFA and LE have a common background, and as previously noted by other authors, their coexistence may not be a coincidence; this could be the result of different responses due to individual genetic predisposition [9]. 
Hydroxychloroquine and chloroquine diphosphate are antimalarial drugs with antilymphocytic effect [18], described as viable treatment options for patients with LE or LPP/FFA. A wide variety of results ranging from good response or stabilization $[5,13,19]$ to treatment failure are reported [17, 20-22]. We deemed our FFA cases active when global photos and/or LPP activity index showed progression during follow-up [13].

Banka et al. [17] observed 2 patients with SLE and FFA, with FFA continuing to deteriorate regardless of the hydroxychloroquine treatment. In our 4 cases, LE appeared years before FFA. The patients had all been using hydroxychloroquine or chloroquine diphosphate by the time they were diagnosed with lupus, even before the diagnosis of FFA. We also observed that this fact did not halt the development or reveal any signs of stabilization of FFA during the use of hydroxychloroquine.
The association of FFA and LE - either discoid or systemic - shows they possibly have a common immune background. The association with other cutaneous and systemic repercussions might be a sign of extensive alopecia and/or a more difficult therapeutic response. In these cases, the response to hydroxychloroquine may be poor.

\section{Statement of Ethics}

The authors have no ethical conflicts to disclose. Patient's consent has been obtained.

\section{Disclosure Statement}

The authors have no conflicts of interest to declare.

\section{References}

1 Olsen EA, Bergfeld WF, Cotsarelis G, et al: Summary of North American Hair Research Society (NAHRS)-sponsored Workshop on Cicatricial Alopecia, Duke University Medical Center, February 10 and 11, 2001. J Am Acad Dermatol 2003;48:103-110.

2 Harries MJ, Paus R: The pathogenesis of primary cicatricial alopecias. Am J Pathol 2010; 177:2152-2162.

3 Kossard S: Postmenopausal frontal fibrosing alopecia. Scarring alopecia in a pattern distribution. Arch Dermatol 1994;130:770-774.

4 Moreno-Ramírez D, Camacho Martínez F: Frontal fibrosing alopecia: a survey in $16 \mathrm{pa}-$ tients. J Eur Acad Dermatol Venereol 2005; 19:700-705.

5 Samrao A, Chew A-L, Price V: Frontal fibrosing alopecia: a clinical review of 36 patients. Br J Dermatol 2010;163:1296-1300.

6 Donati A, Molina L, Doche I, Valente N, Romiti R: Facial papules in frontal fibrosing alopecia. Arch Dermatol 2011;147:14241427.

7 Inaloz HS, Chowdhury MM, Motley RJ: Lupus erythematous/lichen planus overlap syndrome with scarring alopecia. J Eur Acad Dermatol Venereol 2001;15:171-174.

8 Gaffney DC, Sinclair RD, Yong-Gee S: Discoid lupus alopecia complicated by frontal fibrosing alopecia on a background of androge- netic alopecia. Br J Dermatol 2013;169:217218.

9 del Rei M, Pirmez R, Sodré CT, Tosti A: Coexistence of frontal fibrosing alopecia and discoid lupus erythematosus of the scalp in $7 \mathrm{pa}-$ tients: just a coincidence? J Eur Acad Dermatol Venereol 2016;30:151-153.

10 Nagao K, Chen KR: A case of lupus erythematosus/lichen planus overlap syndrome. J Dermatol 2006;33:187-190.

11 Tursen U, Oz O, Ikizoglu G, Kaya TI, Dusmez D: A case of lichen planus-lupus erythematosus overlap syndrome with eyelid involvement. Eur J Ophthalmol 2002;12:244-246.

12 Lospinoso DJ, Fernelius C, Edhegard KD, Finger DR, Arora NS: Lupus erythematosus/ lichen planus overlap syndrome: successful treatment with acitretin. Lupus 2013;22:851854.

13 Chiang C, Sah D, Cho B, Ochoa BE, Price VH: Hydroxychloroquine and lichen planopilaris: efficacy and introduction of Lichen Planopilaris Activity Index scoring system. J Am Acad Dermatol 2010;62:387-392.

14 Miteva M, Tosti A: Treatment options for alopecia: an update, looking to the future. Expert Opin Pharmacother 2012;13:1271-1281.

15 Al-Bari MA: Chloroquine analogues in drug discovery: new directions of uses, mechanisms of actions and toxic manifestations from malaria to multifarious diseases. J Antimicrob Chemother 2015;70:1608-1621.

16 Khan S, Fenton DA, Stefanato CM: Frontal fibrosing alopecia and lupus overlap in a man: guilt by association? Int J Trichology 2013;5: 217-219.

17 Banka N, Mubki T, Bunagan MJ, McElwee K, Shapiro J: Frontal fibrosing alopecia: a retrospective clinical review of 62 patients with treatment outcome and long-term follow-up. Int J Dermatol 2014;53:1324-1330.

18 Kalia S, Dutz JP: New concepts in antimalarial use and mode of action in dermatology. Dermatol Ther 2007;20:160-174.

19 Spencer LA, Hawryluk EB, English JC 3rd: Lichen planopilaris: retrospective study and stepwise therapeutic approach. Arch Dermatol 2009; 145:333-334.

20 Donati A, Assouly P, Matard B, Jouanique C, Reygagne $\mathrm{P}$ : Clinical and photographic assessment of lichen planopilaris treatment efficacy. J Am Acad Dermatol 2011;64:597-598.

21 Racz E, Gho C, Moorman PW, Hegt N, Neumann HAM: Treatment of frontal fibrosing alopecia and lichen planopilaris: a systematic review. J Eur Acad Dermatol Venereol 2013; 27:1461-1470.

22 MacDonald A, Clark C, Holmes S: Frontal fibrosing alopecia: a review of 60 cases. J Am Acad Dermatol 2012;67:955-961. 